\title{
Invitación a Máster en Materiales Nanoestructurados para Aplicaciones Nanotecnológicas (NANOMAT)
}

\author{
Dra. Pilar Cea Mingueza'
}

NANOMAT es un máster oficial de la Universidad de Zaragoza (España) con una duración de 18 meses y está formado por 75 créditos ECTS. El curso es adecuado para licenciados en ciencias, ingeniería, medicina, biología, farmacia y otras titulaciones asimilables, que desean desarrollar carreras en la vanguardia de la nanociencia y nanotecnología.

El curso es eminentemente multidisciplinario y su propósito es proporcionar a los estudiantes un bagaje teórico así como una amplia experiencia práctica y habilidades en la fabricación y caracterización de materiales nanoestructurados y dispositivos con aplicaciones en áreas clave de la nanoquímica, nanofísica y nanobiomedicina.

La Universidad de Zaragoza y los Institutos de Nanociencia y Ciencia de los Materiales de Aragón (INA e ICMA) disponen de un equipamiento excepcional para la fabricación y caracterización de nanomateriales, incluyendo algunos instrumentos únicos en España y Europa.

El curso se imparte completamente en inglés por miembros altamente cualificados del INA, del ICMA y de la Facultad de Ciencias de la Universidad de Zaragoza, y cuenta con la participación de personal de otros centros nacionales e internacionales así como representantes de la industria.

El máster está distribuido en ocho asignaturas (54 créditos ECTS) que incluyen clases teóricas, tutorías, prácticas de laboratorio y casos de estudio. El curso también cuenta con un proyecto fin de máster (21 créditos ECTS). Los estudiantes escogerán el trabajo fin de máster, previa consulta con la coordinadora del curso, atendiendo a su licenciatura de origen y sus propios intereses profesionales y de investigación.

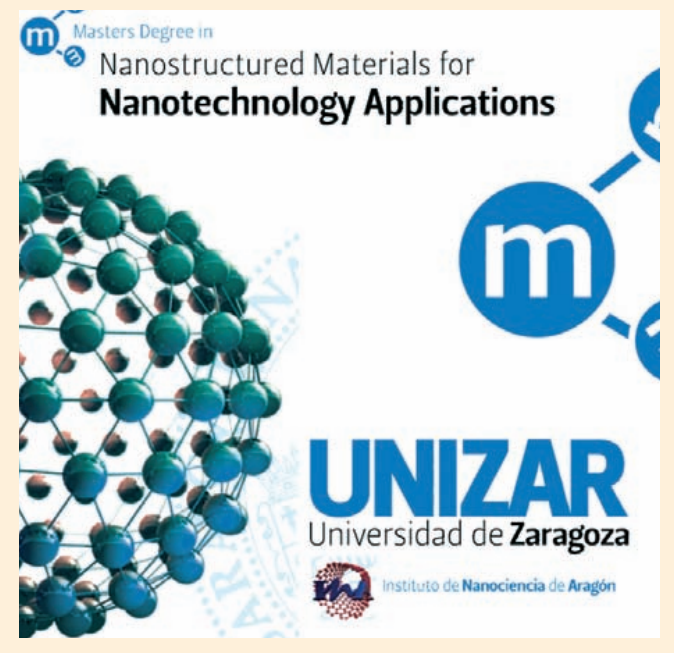

Las asignaturas del curso son:

1. Propiedades fundamentales de los materiales nanoestructurados.

2. Preparación de materiales nanoestructurados.

3. Ensamblaje y caracterización de nanoestructuras.

4. Caracterización I: técnicas químico físicas.

5. Caracterización II: microscopías avanzadas.

6. Fabricación de nanodispositivos y aplicaciones.

7. Casos de estudio para aplicaciones industriales.

\footnotetext{
${ }^{1}$ Investigadora del Área de Química Física de la Facultad de Ciencias, Campus Plaza San Francisco, Universidad de Zaragoza. Coordinadora de NANOMAT.
} 
8. El estudiante elegirá una de las tres asignaturas siguientes:
a. Fenómenos físicos en la nanoescala.
b. Síntesis y procesado de materiales na- noestructurados.
c. Nanobiomedicina.

El máster comienza el 28 de septiembre de 2009. Para más información, puede consultarse la página web del máster: www.unizar.es/nanomat.

Para interesados extranjeros, contactar directamente a la coordinadora del máster al correo: pilarcea@unizar.es o al teléfono +34 976 762296.

9. Trabajo fin de máster. 